\title{
Methylation of MYLK3 gene promoter region: a biomarker to stratify surgical care in ovarian cancer in a multicentre study
}

David L Phelps ${ }^{1,4}$, Jane V Borley ${ }^{1,4}$, Kirsty J Flower ${ }^{1}$, Roberto Dina ${ }^{1}$, Silvia Darb-Esfahani ${ }^{2}$, loana Braicu ${ }^{3}$, Jalid Sehouli ${ }^{3}$, Christina Fotopoulou ${ }^{1,3}$, Charlotte S Wilhelm-Benartzi ${ }^{1}$, Hani Gabra ${ }^{1}$, Joseph Yazbek ${ }^{1}$, Jayanta Chatterjee ${ }^{1}$, Jacey $\mathrm{Ip}^{1}{ }^{1}$, Harun Khan ${ }^{1}$, Marina-Therese Likos-Corbett ${ }^{1}$, Robert Brown ${ }^{1,5}$ and Sadaf Ghaem-Maghami ${ }^{*}, 1,5$

${ }^{1}$ Department of Surgery and Cancer, Imperial College London, Hammersmith Campus, Du Cane Road, London W12 ONN, UK; IInstitute of Pathology, Charité Universitätsmedizin, Berlin 10117, Germany and ${ }^{3}$ Department of Gynaecology, Virchow Campus, Universitätsmedizin, Berlin, Germany

Background: Survival benefit from surgical debulking of ovarian cancer $(O C)$ is well established, but some women, despite total macroscopic clearance of disease, still have poor prognosis. We aimed to identify biomarkers to predict benefit from conventional surgery.

Methods: Clinical data from women debulked for high-stage OC were analysed (Hammersmith Hospital, London, UK; 2001-2014). Infinium's HumanMethylation27 array interrogated tumour DNA for differentially methylated CpG sites, correlated to survival, in patients with the least residual disease (RD; Hammersmith Array). Validation was performed using bisulphite pyrosequencing (Charité Hospital, Berlin, Germany cohort) and The Cancer Genome Atlas' (TCGA) methylation data set. Kaplan-Meier curves and Cox models tested survival.

Results: Altogether 803 women with serous OC were studied. No RD was associated with significantly improved overall survival (OS; hazard ratio (HR) $1.25,95 \% \mathrm{Cl} 1.06-1.47 ; P=0.0076$ ) and progression-free survival (PFS; HR 1.23, 95\% Cl 1.05-1.43; $P=0.012$; Hammersmith database $n=430$ ). Differentially methylated loci within FGF4, FGF21, MYLK2, MYLK3, MYL7, and ITGAE associated with survival. Patients with the least RD had significantly better OS with higher methylation of MYLK3 (Hammersmith ( $\mathrm{HR} 0.51,95 \% \mathrm{Cl}$ 0.31-0.84; $P=0.01$ ), Charité (HR 0.46, 95\% Cl 0.21-1.01; $P=0.05)$, and TCGA (HR 0.64, 95\% Cl 0.44-0.93; $P=0.02)$ ).

Conclusions: MYLK3 methylation is associated with improved OS in patients with the least RD, which could potentially be used to determine response to surgery.

Ovarian cancer (OC) is the most lethal gynaecological malignancy, causing over 125000 deaths per year worldwide (Sankaranarayanan and Ferlay, 2006). Overall survival (OS) is poor due to late presentation, poor surgical outcomes, and the development of chemotherapy resistance. The disease is clinically and molecularly heterogeneous but despite this all patients are treated the same, with cytoreductive surgery and platinum-based chemotherapy (NICE, 2011).

Surgical effort is one of the most important prognostic factors in OC (Agarwal and Kaye, 2003). It is widely accepted that size of residual disease $(\mathrm{RD})$ following surgery influences OS following treatment (Pomel et al, 2008; Shih and Chi, 2010). Five-year OS 
has been shown to improve from 23.9 to 54.6 months in women having surgical resection to no macroscopic RD vs RD $>10 \mathrm{~mm}$ (Wimberger, 2010). As surgical expertise and effort have improved total macroscopic clearance rates, it has become apparent that some patients still respond poorly to treatment, despite complete removal of all macroscopic tumour at primary surgery.

Gene expression signatures are associated with different survival outcomes in high-grade serous OC (HGSOC) patients (Tothill et al, 2008; TCGARN, 2011). Prognostic biomarkers associated with survival in HGSOC have been widely investigated in the literature (Fiegl et al, 2008; Flanagan et al, 2013). Notable examples include overexpression of individual genes such as VEGF, HER2 and progesterone receptor, which have been associated with progression-free survival (PFS) and OS (Yu et al, 2013; Zhao et al, 2013). Differential DNA methylation at CpG sites, particularly at CpG islands in gene promoter regions, is also associated with PFS and OS of HGSOC patients (Dai et al, 2011, 2013; Wang et al, 2014; Chou et al, 2015). These findings improve our understanding of specific tumour biology and raise the possibility of utilising these biological characteristics to allow patient stratification to treatment. Differential methylation of O-6-methylguanine-DNA methyltransferase is already in use clinically to stratify treatment in those with malignant glioblastomas (Hegi et al, 2005; Malmstrom et al, 2012; Gupta et al, 2015).

To our knowledge there are no current biomarkers that can be used to identify women with HGSOC who do poorly despite optimal surgical treatment (Borley et al, 2012). Thus, there is a proportion of these patients who undergo maximal effort radical surgical treatment, with all of the associated potential morbidity and mortality, without the associated survival benefit. For women who have a poor prognosis, despite maximal surgical reduction in disease, alternative therapeutic approaches would need to be applied in a context of individualised surgical treatment.

The aim of this study was to identify the proportion of women that have poor prognosis despite maximal surgical effort. Also we aimed to identify and validate potential DNA methylation biomarkers, which may enable stratification of care to different surgical and treatment pathways. DNA methylation as a biomarker was selected due to its relative stability ex vivo and in vivo, as well as the future potential to detect DNA methylation changes noninvasively in circulating tumour DNA (Keeley et al, 2013; Bettegowda et al, 2014). As the success of surgical debulking is associated with the ability of tumour cells to invade and metastasise, we focused on the 'focal-adhesion' and 'adherensjunction' pathways, involved with cell surface adhesion, and the 'regulation of actin cytoskeleton' pathway involved with cell migration (Laboratories, 2013).

\section{PATIENTS AND METHODS}

This proposal was reviewed and agreed by the 'HTA-approved' Imperial College Healthcare NHS Trust Tissue Bank (authorised by the Wales MREC) project reference Gyn_HG_12_060 and Gyn_HG_13_020. Written consent was obtained from all patients included in this study who provided tumour tissue for research. Reporting recommendations for tumour marker (REMARK) criteria were followed throughout this study.

Historical Hammersmith database. Electronic patient records from January 2001 to December 2014 at Hammersmith Hospital, Imperial College Healthcare NHS Trust (ICHNT; West London Gynaecology Oncology Surgical Centre) were searched to identify cases. Inclusion criteria were primary serous ovarian, fallopian, or peritoneal carcinoma (mixed tumours were included if the epithelial component predominated), stage 3/4, and primary surgical debulk was initial treatment. Staging was classified using the version of International Federation of Gynaecology and Obstetrics staging classifications in place at the time of diagnosis. OS and PFS were determined from the date of surgery until the date of the event (death or recurrence/progression, respectively) or last known contact. All survival data were updated between 15 June 2015 and 03 July 2015. If patients were lost to follow-up they were censored accordingly. RD status was classified as total debulking ( $0 \mathrm{~mm} \mathrm{RD})$, optimal debulking ( $\mathrm{RD} \leqslant 10 \mathrm{~mm}$ ), and suboptimal debulking ( $>10 \mathrm{~mm} \mathrm{RD)}$.

Patient tumour samples. Fresh-frozen OC tissue was collected and stored at $-80^{\circ} \mathrm{C}$ by ICHNT Biobank London and Tumour Bank Ovarian Cancer (TOC) (www.toc-network.de) at Charité Hospital Berlin. Tumours were derived from primary debulking surgery and were stage $3 / 4$ serous histology. Tumour sample quality was ensured by histopathology (excluded if tumour cell nuclei $<20 \%$ ). Overall survival and PFS were determined from the date of surgery until the date of the event (death or progression, respectively) or last known contact and censored if lost to followup. The Hammersmith and TOC-Charite DNA methylation data sets included serous tumour with similarly matched age and stage. Residual disease differed between the data sets, with suboptimal debulking rates lower in the TOC-Charité and The Cancer Genome Atlas (TCGA) data sets compared to Hammersmith. This may reflect the significant variability between surgeons' approach to radicality, skills, and the significant difficulty in the reproducibility of tumour measurements, which corresponds with previously described variability among surgical expertise and outcomes among different centres and different countries (Chi et al, 2009; Vergote et al, 2010). The potential effect this may have on survival was mitigated by adjusting for $\mathrm{RD}$ as a confounder. Of all patients that were fit to have chemotherapy, over 95\% received platinum-based chemotherapy.

TCGA data set. Publicly available clinical, surgical, methylation, and expression data for patients with serous cystadenocarcinoma were downloaded from TCGA data portal (https://tcga-data.nci. nih.gov/tcga/; TCGARN, 2011). Methylation data were obtained using $27 \mathrm{k}$ and 450k Illumina Infinium (San Diego, CA, USA) methylation arrays. We included 277 patients with stage 3/4 disease. Supplementary Table S1 shows the clinical characteristics of the historical Hammersmith database cases and the three independent methylation data sets.

Tumour DNA extraction and bisulphite conversion. Up to $500 \mathrm{mg}$ of tumour tissue was used per sample, and DNA was extracted using the chlorinated Nucleon extraction (GE Healthcare Life Sciences, Buckinghamshire, UK) method. Tumour DNA was bisulphite-converted with EZ-96 DNA Methylation Kit (Zymo, Irvine, CA, USA) as per the manufacturer's protocol. A unit of $1 \mu \mathrm{g}$ of genomic DNA was used for each sample.

Illumina Infinium HumanMethylation27 beadchip array. Discovery-set samples (Hammersmith array data set) were processed as per the Infinium Assay Methylation Protocol (Illumina). A unit of $200 \mathrm{ng}$ of genomic DNA was used for bisulphite conversion. Methylation data were summarised as $\beta$-values, calculated as $M /(M+U)$, where $M$ is the signal from methylated beads and $U$ is the signal from unmethylated beads at the targeted $\mathrm{CpG}$ site. $\beta$-values were adjusted against background noise and data were logtransformed to achieve a normal distribution. Probes with a detection $P$-value of $>0.05$ were removed. Quality control checks were performed with GenomeStudio (Illumina).

Pyrosequencing. DNA methylation of the validation-set samples (TOC-Charité data set) was determined through PCR amplification with biotinylated primers (Invitrogen Life Sciences, Carlsbad, CA, USA; and Sigma Aldrich, St Louis, MO, USA) using Pyromark Assay Design Software version 2.0 (Supplementary Table S2), and 
Qiagen (Hilden, Germany) Pyromark Q96 MD pyrosequencer as previously described (Dai et al, 2013). Amplified products were confirmed with agarose gel electrophoresis. Pyro-Q-CpG (Qiagen) software was used to calculate $\mathrm{CpG}$ site methylation values using the same $M /(M+U)$ calculation as with the beadchip array.

Statistical analysis. Analysis was performed using R v3.2.2. Univariate analyses using the log-rank test were performed and corresponding log-rank $P$-values reported. The OS and PFS analyses were plotted using Kaplan-Meier survival curves. Patients lost to follow-up were censored and their last known contact used for survival purposes. Multivariable adjustment was performed for true confounders, including age, stage, grade, RD status, and batch using Cox proportional hazards models and shown as multivariable Cox $P$-values. Hazard ratios (HR) and 95\% confidence intervals (95\% CIs) are reported alongside the Cox $P$-values. Statistical significance was set at $P<0.05$.

\section{RESULTS}

Changing surgical trends towards more radical surgery are associated with improved survival. Over the past 15 years, surgical trends have moved towards a more radical approach in response to mounting evidence of prognostic benefit with reduced tumour burden (Chi et al, 2009). To evaluate this trend at Hammersmith Hospital and to establish whether this impacted upon survival, debulking rates were compared year by year and survival of these patients was evaluated using Kaplan-Meier survival curves; Figure 1. In 2013 and 2014, less than a quarter of patients had any measurable disease left after surgery, compared with over $75 \%$ in 2001-2003 (Figure 1A). A total of 430 women who underwent primary debulking surgery were included in the analyses. The survival curves in Figure $1 \mathrm{~B}$ and $\mathrm{C}$ show statistically significant OS (HR 1.25, 95\% CI 1.04, 1.47) and PFS (HR 1.23, 95\% CI $1.05,1.43$ ) advantage for women with the least RD after surgery. Median OS for patients with $\mathrm{RD}>10 \mathrm{~mm}$ was 13.2 months, compared to those with zero RD with median OS of 26.2 months. Median PFS (Figure 1C) improved with reducing RD as expected.

Despite patients receiving total macroscopic clearance, it is clear that some of these patients, even with maximal surgical effort, do poorly with respect to survival. Of all totally debulked (0 mm RD) patients in the Hammersmith database, 8.8\% died within 12 months of their surgery. Median OS in this group of women was only 3 months (inter-quartile range $0.5-8.0$ months). These patients may not have benefitted from extensive surgery and may have potentially benefitted from alternative treatment strategies, highlighting the need for a robust biomarker to identify these women. We therefore used three independent tumour DNA data sets to identify and validate DNA methylation biomarkers associated with survival in patients with the least RD.

DNA methylation biomarkers predict poor survival in optimally debulked patients. To identify differential methylation associated with survival in optimally debulked patients, DNA methylation of tumour DNA determined through Illumina's Infinium HumanMethylation27 BeadChip array was investigated (Hammersmith array data set). Multivariable Cox proportional hazards models were performed on optimally debulked patients only. The Kyoto Encyclopaedia of Genes and Genomes was used to identify 732 methylation probes covering genes involved with adhesion and migration pathways. Our hypothesis was that genes involved in this pathway could affect survival by increasing metastatic potential and tumour invasiveness, therefore leading to biologically more
A

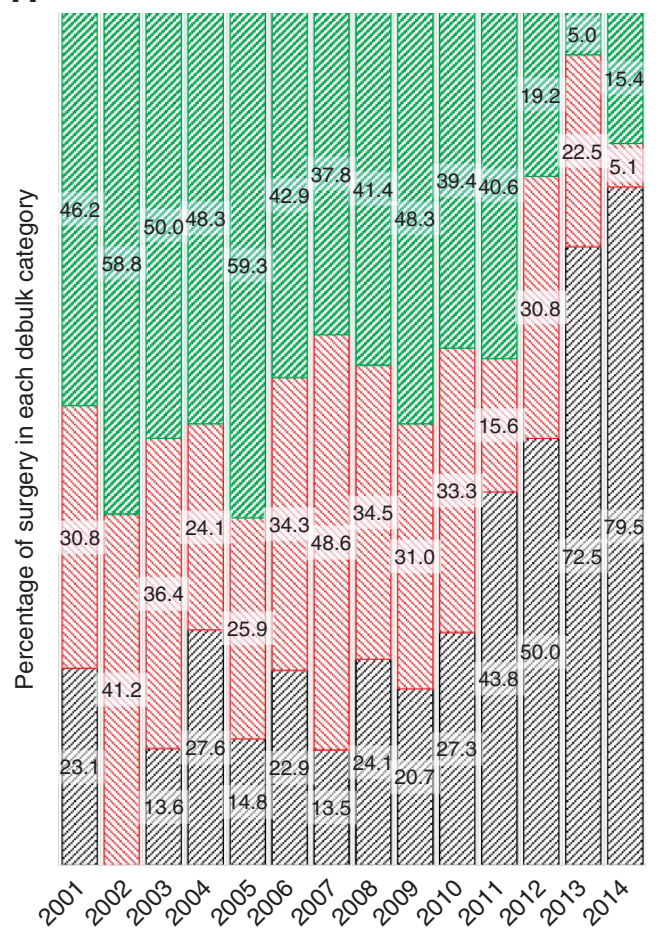

B

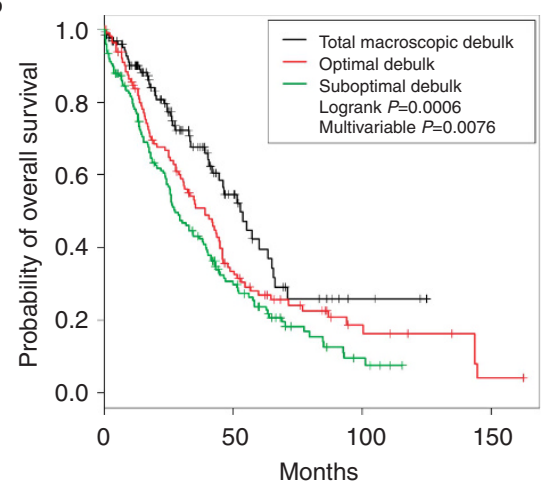

C

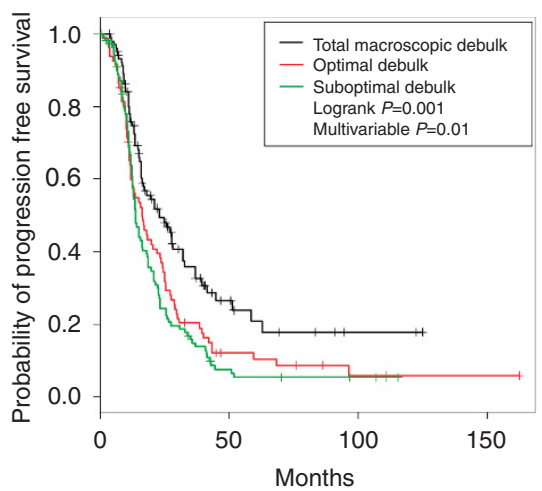

Figure 1. Surgical trends and survival according to debulk status in the Hammersmith Database between 2001 and 2014 . (A) Debulk rates (percentage of all cases) at Hammersmith Hospital between 2001 and 2014. Total macroscopic debulk (0 mm RD) shown in black, optimal debulk (RD 1-10 mm) in red, and suboptimal debulk (RD>10 mm) in green. (B) Overall survival - total debulk vs suboptimal debulk (HR=1.25 (95\% Cl $1.06,1.47)$ ). (C) Progression-free survival - total debulk vs suboptimal debulk (HR=1.23 (95\% Cl 1.05, 1.43)). Multivariable Cox proportional hazards model adjusting for age, stage, grade, and histology. 
aggressive tumours. The hypothesis-driven analysis was performed on 732 loci and 65 were found to show differential methylation associated with OS $(P<0.05)$. To increase the likelihood of detecting clinically relevant differential methylation we chose a differential methylation cut-off of $20 \%$. Consequently, 36 probes were included in further analysis. Cox proportional hazards models were used to

Table 1. Six most significant differentially methylated loci in Hammersmith discovery cohort using $27 \mathrm{k}$ methylation array associated with survival in patients with the least residual disease (optimal debulk $n=39$ )

\begin{tabular}{|l|l|c|c|c|c|}
\hline Probe ID & $\begin{array}{l}\text { Gene } \\
\text { name }\end{array}$ & $\begin{array}{c}\text { Median } \\
\text { methylation } \\
\%\end{array}$ & $\begin{array}{c}\text { Cox } \\
\text { model } \\
(\boldsymbol{P})^{\mathbf{a}}\end{array}$ & HR & 95\% Cl \\
\hline cg14578030 & FGF4 & 85.2 & 0.011 & 0.39 & $0.19,0.81$ \\
\hline cg21856603 & ITGAE & 77.5 & 0.042 & 0.48 & $0.24,0.98$ \\
\hline cg16155702 & FGF21 & 74.3 & 0.031 & 0.50 & $0.26,0.94$ \\
\hline cg13247990 & MYLK3 & 80.1 & 0.008 & 0.51 & $0.31,0.84$ \\
\hline cg19961522 & MYLK2 & 73.2 & 0.024 & 0.57 & $0.35,0.93$ \\
\hline cg23370883 & MYL7 & 61.7 & 0.026 & 0.57 & $0.35,0.94$ \\
\hline f & & & & &
\end{tabular}

Abbreviations: $\mathrm{Cl}=$ confidence interval; $F G F 4=$ fibroblast growth factor 4; FGF21= fibroblast growth factor 21; HR= hazards ratio; ITGAE = integrin alpha $\mathrm{E} ; \mathrm{MYLK2}=$ myosin light chain kinase 2; MYLK3= myosin light chain kinase 3; MYL7=myosin light chain 7, regulatory.

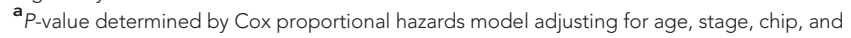
residual disease status. Supplementary Table S3 lists the multivariable Cox proportional hazards survival analysis for the top 27 significant probes. adjust for age, stage, chip, and $\mathrm{RD}$ to investigate whether patients had different survival distributions per locus. Of the 36 loci, 27 were found to be significant (Supplementary Table S3). The six probes with the largest effect size, FGF4, FGF21, MYLK2, myosin light chain kinase 3 (MYLK3), MYL7, and ITGAE are shown in Table 1, and were taken forward for validation.

MYLK3 CpG locus methylation predicts poor outcome in patients with the least RD. Validation of the six significant loci associated with survival in the Hammersmith Array data set was performed using an independent data set (TOC-Charité). DNA methylation of all six loci in OC tumour DNA was quantified by bisulphite pyrosequencing of the TOC-Charité data set validation cohort. As there was a low number of suboptimally debulked patients in this group $(n=6)$, total debulk $(n=47)$ was compared to any amount of $\mathrm{RD}(n=36)$. MYLK3 was the only locus to validate in TOC-Charité $(P=0.025$; Figure $2 \mathrm{~B})$. The same analysis was performed in TCGA data set for all six discovery loci (Supplementary Table S4). Although MYLK3 did not quite reach significance $(P=0.06)$, in the TCGA analysis there is a clear trend in the survival curves (Figure $2 \mathrm{~A}-\mathrm{C}$ ) towards improved survival with increased methylation of MYLK3 in all data sets. Survival for patients with low methylation appears to be comparable to that of their suboptimally debulked counterparts, who had significant RD. Patients appeared not to benefit from the radical surgery in the same way their highly methylated counterparts did.

Further multivariable analysis focused only on patients with the least $\mathrm{RD}$, as clinically it would be beneficial to be able to predict
A

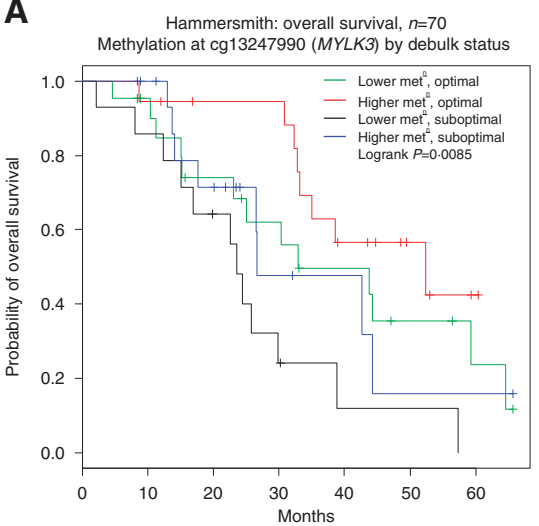

D

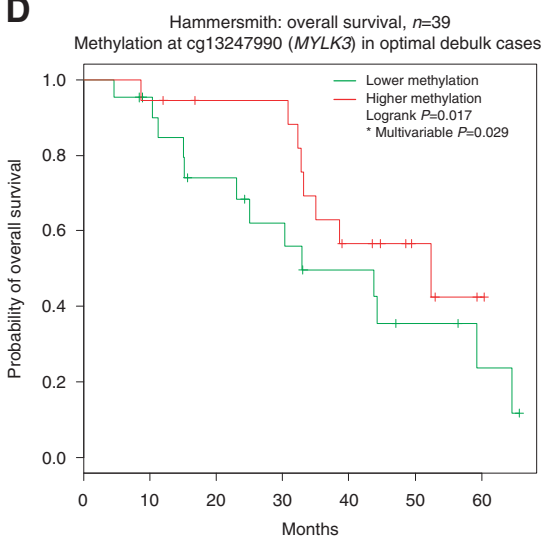

B

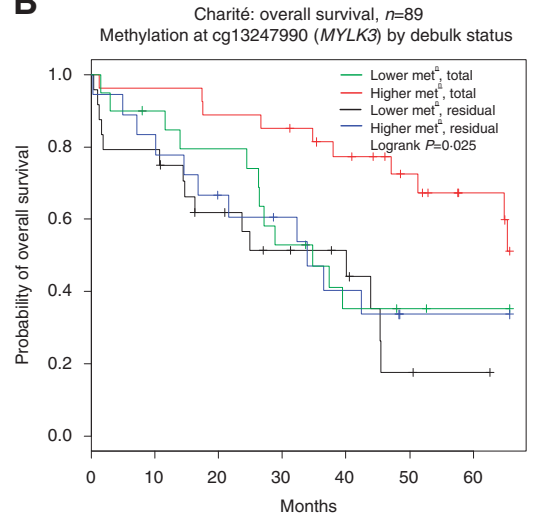

E

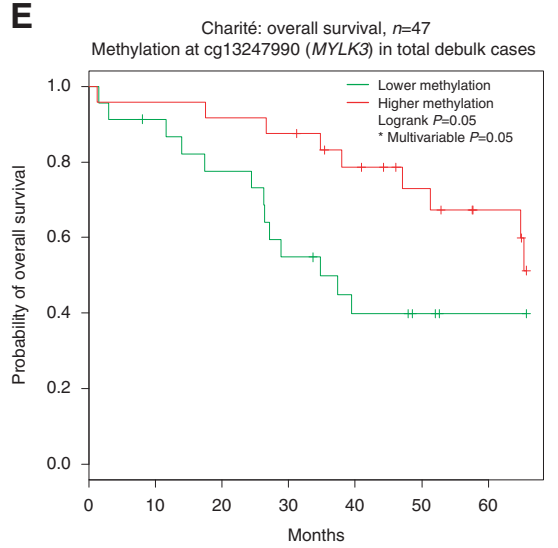

C

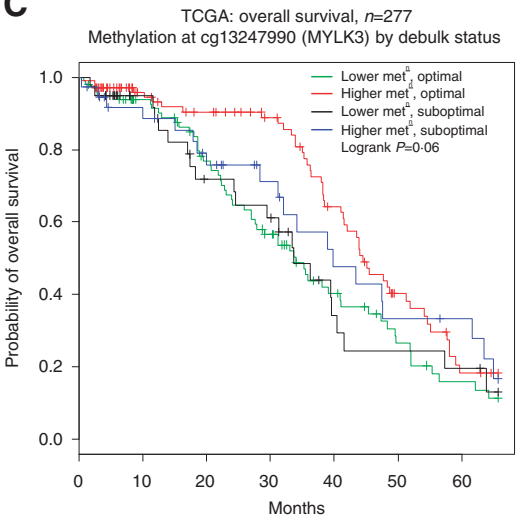

$\mathbf{F}$

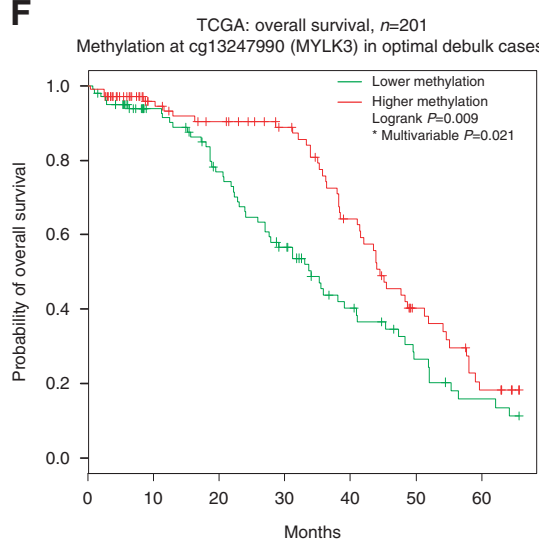

Figure 2. Kaplan-Meier overall survival curves according to debulk status and methylation $\left(\operatorname{met}^{\mathrm{n}}\right)$, shown as higher or lower than the median methylation for all patients. (A) Probe cg13247990 on 27k methylation array in discovery cohort (Hammersmith Array data set) with debulk status shown as 'optimal' vs 'suboptimal'. (B) Pyrosequencing of MYLK3 locus in validation cohort (Charité data set) with debulk status shown as 'total' debulk vs any 'residual' disease. (C) Probe cg13247990 on methylation array in TCGA data set with debulk shown as 'optimal' vs 'suboptimal'. (D) Hammersmith patients with optimal debulk $(\mathrm{HR}=0.51(95 \% \mathrm{Cl} 0.31,0.84)$. (E) Charite patients with total debulk $(\mathrm{HR}=0.46(95 \% \mathrm{Cl} 0.21,1.01))$. (F) TCGA patients with optimal debulk $(\mathrm{HR}=0.64(95 \% \mathrm{Cl} 0.44,0.93)$. *All multivariable analyses adjusted for confounders age and stage plus grade, chip, and RD status when appropriate. 
Table 2. Six candidate loci validation analysis in the Charité cohort using bisulphite pyrosequencing of differentially methylated loci associated with survival in patients with the least residual disease (total debulk $n=47$ )

\begin{tabular}{|c|c|c|c|c|c|}
\hline Probe ID & $\begin{array}{l}\text { Gene } \\
\text { name }\end{array}$ & $\begin{array}{c}\text { Median } \\
\text { methylation } \\
\%\end{array}$ & $\begin{array}{l}\text { Cox } \\
\text { model } \\
(P)^{a}\end{array}$ & HR & $95 \% \mathrm{Cl}$ \\
\hline cg14578030 & FGF4 & 92.2 & 0.355 & 0.68 & $0.30,1.55$ \\
\hline cg21856603 & ITGAE & NA & NA & NA & NA \\
\hline cg16155702 & FGF21 & 78.4 & 0.064 & 0.44 & $0.18,1.05$ \\
\hline cg13247990 & MYLK3 & 86.3 & 0.053 & 0.51 & $0.21,1.01$ \\
\hline cg19961522 & MYLK2 & 90.5 & 0.998 & 1.00 & $0.43,2.30$ \\
\hline cg23370883 & MYL7 & 77.0 & 0.224 & 0.59 & $0.26,1.38$ \\
\hline \multicolumn{6}{|c|}{$\begin{array}{l}\text { Abbreviations: } \mathrm{Cl}=\text { confidence interval; } F G F 4=\text { fibroblast growth factor } 4 ; F G F 21= \\
\text { fibroblast growth factor } 21 ; \mathrm{HR}=\text { hazards ratio; ITGAE =integrin alpha } E ; M Y L K 2=\text { myosin } \\
\text { light chain kinase } 2 ; M Y L K 3=\text { myosin light chain kinase } 3 ; M Y L 7=\text { myosin light chain } 7 \\
\text { regulatory; } N A=\text { not available. ITGAE results not available as pyrosequencing assay failed. } \\
\text { a }_{P \text {-value determined by Cox proportional hazards model adjusting for age, stage, anc }} \\
\text { grade. }\end{array}$} \\
\hline
\end{tabular}

which patients would benefit most from radical surgery. Differential methylation survival analysis of the MYLK3 locus was conducted in the Hammersmith and TOC-Charite data sets, as well as TCGA data set (Table 2 and Supplementary Table S4). Higher methylation of the MYLK3 locus is associated with OS in patients with the least RD in all three independent cohorts of patients (Figure 2D and E), however the TOC-Charite data set was borderline significant $(P=0.053)$.

The optimally debulked Hammersmith patients with higher methylation at the MYLK3 locus had median OS of 52.4 months, falling to 33.0 months in patients with lower methylation. Patients with higher methylation in TCGA data set had median OS of 44.5 months, a survival advantage of 10.5 months when compared to median OS of the low-methylation group, which was 34.0 months. Charite patients had the largest survival advantage from higher methylation in their totally debulked patients with median OS of 68.0 months with higher methylation, falling to 34.7 months in the lower-methylation group. On average, women with higher methylation of the MYLK3 locus appear to have 10.5-33.2 months' additional OS advantage across the three independent cohorts in this study. The TOC-Charite result may reflect that the effect of higher methylation at MYLK3 is most profound in patients with total macroscopic clearance of their disease. To further investigate this theory, all three methylation data sets were combined, totalling 436 women for analysis in a similar manner to the TOC-Charité and TCGA validation analyses. The combination of all data sets increases the numbers of patients in all subgroups and enables an analysis of the entire cohort splitting the groups into 'total macroscopic clearance of disease' and 'any RD'. Potential batch effects, combining these data sets, were adjusted for in the multivariable Cox model for this analysis as an indicator variable. Figure 3 shows a highly significant association (log-rank $P=4.33 \mathrm{e}^{-7}$, Cox $P=0.0040$, HR 0.67, 95\% CI 0.51, 0.88) between OS and methylation of MYLK3, most prominently in totally debulked patients. Patients with any RD also appear to gain significant survival benefit by having higher methylation of MYLK3. The protective benefit of higher MYLK3 methylation pulls the survival curve for the RD group into a similar position to the totally debulked patients with low methylation and appears to be a protective factor itself.

Expression of MYLK3 is not directly linked to survival or methylation of the MYLK3 locus. Hammersmith and TOCCharité data sets did not have matched expression data, so TCGA expression data set was interrogated separately. All 277 patients in TCGA data set who had methylation data had matched expression

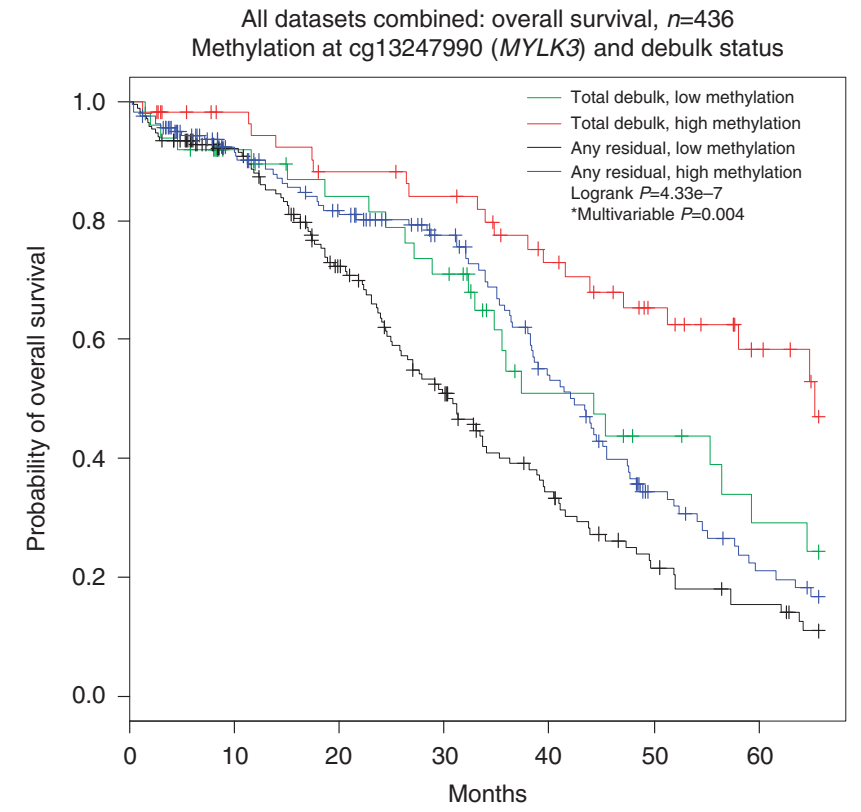

Figure 3. Overall survival in all three data sets combined. Overall survival significantly improved by reducing RD and increasing MYLK3 methylation. Survival benefit from total debulk appears to be lost in the presence of low methylation of MYLK3. Similarly, women with RD gain survival from having high MYLK3 methylation. Total of 436 women included from Hammersmith, Charité, and TCGA data sets. Survival dichotomised by median methylation of MYLK3 derived from the total cohort (84.9\%) and debulk status. *Multivariable model adjusted for age, stage, grade, debulk, batch, and an integer variable to adjust for combining data sets.

data for MYLK3. Potential correlation between methylation and expression was investigated with Spearman's rank correlation test (Figure 4A). There was no significant correlation between methylation of the MYLK3 locus and expression of the MYLK3 gene $(P=0.55, \rho=-0.042)$. Furthermore, there was no statistically significant association between expression of the gene and OS (log-rank $P=0.143$, Cox $P=0.152$; Figure $4 \mathrm{~B})$. The same analysis was also performed on the other five discovery genes, all of which similarly showed no association with survival (data not shown).

\section{DISCUSSION}

Currently, all patients with advanced stage OC are treated with surgery and chemotherapy, despite our expanding knowledge of the heterogeneity of the disease. The benefit of achieving total macroscopic clearance of disease is now well recognised and these data show that this has served patients well in the past, as survival is significantly improved by performing increasingly radical surgery. However, although this blanket approach improves survival across a population, it takes no account of individual tumour biology and therefore is not necessarily the best treatment for all patients. Increasingly, more is known about the biology of tumours, chemotherapy evasion, the development of resistance, and the process of metastasis: future treatments will increasingly be focused on exploiting these unique characteristics. In the new age of personalised medicine, where patients should expect to have their care tailored for their individual needs, it is essential to determine biomarkers that will allow the stratification of care and to truly personalise medicine. Surgical biomarker studies tend to have poor surgical annotations, and most studies are limited by heterogeneous samples and lack adjustment for major confounding factors (Borley et al, 2012). Clinical, histological, and surgical 

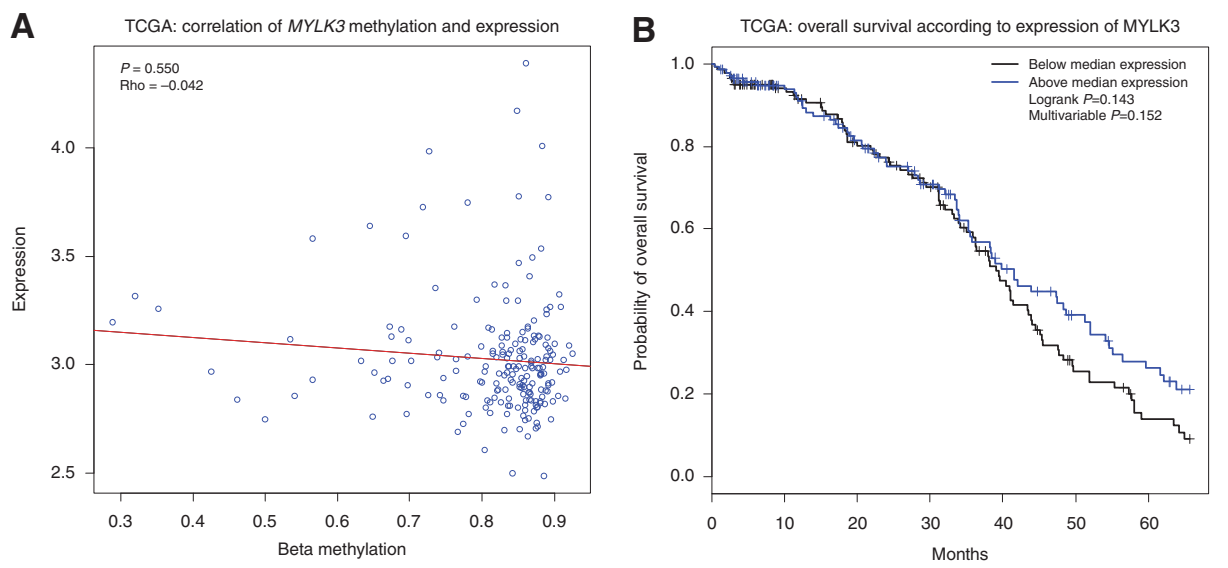

Figure 4. Expression correlations with survival and methylation of MYLK3. (A) Correlation plot of MYLK3 expression and methylation showing no correlation (Pearson's $\rho-0.042, P=0.550$ ) between the methylation level of MYLK3 and expression of the gene. (B) Kaplan-Meier survival curve showing no significant relationship between MYLK3 expression and OS (log-rank $P=0.156$, Cox $P=0.079(\mathrm{HR} 0.70,95 \% \mathrm{Cl} 0.47,1.04)$ ).

annotations in this study were collected to a very high standard, mitigating some of the limitations inherent in previous surgical biomarker research.

MYLK3 is a protein-coding gene found on chromosome 16 (16q11.2) recognised for its role in the regulation of actin cytoskeleton and immune response signalling. It phosphorylates cardiac myosin heavy (MYH7B) and light (MYL2) chains, potentiating the force and rate of cross bridge recruitment in myocytes (Chan et al, 2008). Also, myosin light chain kinases have been linked to the regulation of epithelial cell survival, and knockdown of the gene has led to apoptosis of epithelial breast line cells in vitro (Connell and Helfman, 2006). Myosin light chain kinase on chromosome 3, has a role in epithelial tight junction permeability, has been shown to be a warning marker for gastric cancer and has also been shown to promote cell proliferation (Al-Sadi et al, 2008; Han et al, 2011; Chen et al, 2012). It is not clear why differential methylation at this CpG locus in MYLK3 seems to affect $O S$ in patients who have received maximal surgical effort. Our findings may represent the OC cells' ability to invade their basement membrane, move across epithelial junctions, and/or have a role in metastasis. To our knowledge there is no current evidence showing any association between DNA methylation of the MYLK3 gene promoter region and expression of the gene. Nor is there any literature linking MYLK3 to OC outcomes. Differential $\mathrm{CpG}$ island methylation is often associated with gene expression, but MYLK3 does not contain a regulatory CpG island in its promoter region. Differential methylation can however affect a locus outside a $\mathrm{CpG}$ island by influencing transcription factor binding (Medvedeva et al, 2014). The locus examined here sits within the promoter region of the gene, only 45 base pairs from the transcription start site, but not as part of a $\mathrm{CpG}$ island. It is therefore not likely that methylation at the locus would have a direct effect on expression of MYLK3 specifically. An alternative hypothesis is that methylation at this locus could be a surrogate marker for effects on the transcription of other genes. Further work should be performed to establish the exact nature, cause, and effect of this differential methylation on OC survival. In the meantime, differential methylation at the cg13247990 MYLK3 locus appears to have significant potential as a surgical biomarker, which may enable stratification of surgical care according to the biology of patients' tumours. The MYLK3 biomarker could potentially be measured from tumour biopsy samples or from cell-free circulating tumour DNA (Bettegowda et al, 2014). Those patients with high methylation appear to have the best outcome from total macroscopic debulking and could therefore be assigned the most radical surgery. Women with low methylation may not benefit from radical upfront surgery, and other treatment strategies may be more beneficial, including the consideration of neoadjuvant chemotherapy. Clearly, the potential benefits of our findings would need to be confirmed in an appropriately controlled clinical trial.

In the Hammersmith database cohort of 430 women, $8.8 \%$ of totally debulked patients had progressed or died within 12 months of surgery, with very poor median OS of 3 months. It is questionable how much benefit these women received from radical surgery and our conventional treatment approaches. Differential methylation of MYLK3 could potentially have an exciting part in the future of personalised medicine in OC surgery. The ability to determine which women will respond well to radical surgery would be a significant advancement in the treatment of OC.

\section{ACKNOWLEDGEMENTS}

Special thanks to patients at ICHNT and Charité who kindly provided samples. Cancer Research UK Imperial Centre funded the clinical research fellows (DLP/JVB). The research was funded/ supported by the National Institute for Health Research Imperial Biomedical Research Centre, the Imperial Experimental Cancer Medicine Centre and the Cancer Research UK Imperial Centre at Imperial College London, and ICHNT. Special thanks to Naina Patel and Nona Rama for their support with sample collection and archiving.

${ }^{4}$ These authors contributed equally to this work.

${ }^{5}$ These senior authors contributed equally to this work.

\section{CONFLICT OF INTEREST}

IB has honoraria from Fujirebio, Myriad Genetics, Roche, Glycotype $\mathrm{GmbH}$, and AstraZeneca, and consulting or advisory roles with Glycotype and Fujirebio. She has received research funding from Takeda, Angle, and AstraZeneca, and travel, accommodation, or expenses payments from GlaxoSmithKline, Amgen, Essex Pharma, Fujirebio, and Roche. The remaining authors declare no conflict of interest.

\section{REFERENCES}

Agarwal R, Kaye SB (2003) Ovarian cancer: strategies for overcoming resistance to chemotherapy. Nat Rev Cancer 3(7): 502-516. 
Al-Sadi R, Ye D, Dokladny K, Ma TY (2008) Mechanism of IL-1beta-induced increase in intestinal epithelial tight junction permeability. J Immunol 180(8): 5653-5661.

Bettegowda C, Sausen M, Leary RJ, Kinde I, Wang Y, Agrawal N, Bartlett BR, Wang H, Luber B, Alani RM, Antonarakis ES, Azad NS, Bardelli A, Brem H, Cameron JL, Lee CC, Fecher LA, Gallia GL, Gibbs P, Le D, Giuntoli RL, Goggins M, Hogarty MD, Holdhoff M, Hong SM, Jiao Y, Juhl HH, Kim JJ, Siravegna G, Laheru DA, Lauricella C, Lim M, Lipson EJ, Marie SK, Netto GJ, Oliner KS, Olivi A, Olsson L, Riggins GJ, Sartore-Bianchi A, Schmidt K, Shih LM, Oba-Shinjo SM, Siena S, Theodorescu D, Tie J, Harkins TT, Veronese S, Wang TL, Weingart JD, Wolfgang CL, Wood LD, Xing D, Hruban RH, Wu J, Allen PJ, Schmidt CM, Choti MA, Velculescu VE, Kinzler KW, Vogelstein B, Papadopoulos N, Diaz Jr. LA (2014) Detection of circulating tumor DNA in early- and late-stage human malignancies. Sci Transl Med 6(224): 224ra24.

Borley J, Wilhelm-Benartzi C, Brown R, Ghaem-Maghami S (2012) Does tumour biology determine surgical success in the treatment of epithelial ovarian cancer? A systematic literature review. Br J Cancer 107(7): 1069-1074.

Chan JY, Takeda M, Briggs LE, Graham ML, Lu JT, Horikoshi N, Weinberg EO, Aoki H, Sato N, Chien KR, Kasahara H (2008) Identification of cardiac-specific myosin light chain kinase. Circ Res 102(5): 571-580.

Chen L, Su L, Li J, Zheng Y, Yu B, Yu Y, Yan M, Gu Q, Zhu Z, Liu B (2012) Hypermethylated FAM5C and MYLK in serum as diagnosis and pre-warning markers for gastric cancer. Dis markers 32(3): 195-202.

Chi DS, Eisenhauer EL, Zivanovic O, Sonoda Y, Abu-Rustum NR, Levine DA, Guile MW, Bristow RE, Aghajanian C, Barakat RR (2009) Improved progression-free and overall survival in advanced ovarian cancer as a result of a change in surgical paradigm. Gynecol Oncol 114(1): 26-31.

Chou JL, Huang RL, Shay J, Chen LY, Lin SJ, Yan PS, Chao WT, Lai YH, Lai YL, Chao TK, Lee CI, Tai CK, Wu SF, Nephew KP, Huang TH, Lai HC, Chan MW (2015) Hypermethylation of the TGF-beta target, ABCA1 is associated with poor prognosis in ovarian cancer patients. Clin Epigenetics 7(1): 1.

Connell LE, Helfman DM (2006) Myosin light chain kinase plays a role in the regulation of epithelial cell survival. J Cell Sci 119(Pt 11): 2269-2281.

Dai W, Teodoridis JM, Zeller C, Graham J, Hersey J, Flanagan JM, Stronach E, Millan DW, Siddiqui N, Paul J, Brown R (2011) Systematic CpG islands methylation profiling of genes in the wnt pathway in epithelial ovarian cancer identifies biomarkers of progression-free survival. Clin Cancer Res 17(12): 4052-4062.

Dai W, Zeller C, Masrour N, Siddiqui N, Paul J, Brown R (2013) Promoter $\mathrm{CpG}$ island methylation of genes in key cancer pathways associates with clinical outcome in high-grade serous ovarian cancer. Clin Cancer Res 19(20): 5788-5797.

Fiegl H, Windbichler G, Mueller-Holzner E, Goebel G, Lechner M, Jacobs IJ, Widschwendter M (2008) HOXA11 DNA methylation-a novel prognostic biomarker in ovarian cancer. Int J Cancer 123(3): 725-729.

Flanagan JM, Wilhelm-Benartzi CS, Metcalf M, Kaye SB, Brown R (2013) Association of somatic DNA methylation variability with progression-free survival and toxicity in ovarian cancer patients. Ann Oncol 24(11): 2813-2818.

Gupta SK, Kizilbash SH, Carlson BL, Mladek AC, Boakye-Agyeman F, Bakken KK, Pokorny JL, Schroeder MA, Decker PA, Cen L, Eckel-Passow JE, Sarkar G, Ballman KV, Reid JM, Jenkins RB, Verhaak RG, Sulman EP, Kitange GJ, Sarkaria JN (2015) Delineation of MGMT hypermethylation as a biomarker for veliparib-mediated temozolomide-sensitizing therapy of glioblastoma. J Natl Cancer Inst 108(5): 1-10.

Han YJ, Ma SF, Yourek G, Park YD, Garcia JG (2011) A transcribed pseudogene of MYLK promotes cell proliferation. FASEB J 25(7): 2305-2312.

Hegi ME, Diserens AC, Gorlia T, Hamou MF, de Tribolet N, Weller M, Kros JM, Hainfellner JA, Mason W, Mariani L, Bromberg JE, Hau P, Mirimanoff RO, Cairncross JG, Janzer RC, Stupp R (2005) MGMT gene silencing and benefit from temozolomide in glioblastoma. N Engl J Med 352(10): 997-1003.

Keeley B, Stark A, Pisanic Ii TR, Kwak R, Zhang Y, Wrangle J, Baylin S, Herman J, Ahuja N, Brock MV, Wang T-H (2013) Extraction and processing of circulating DNA from large sample volumes using methylation on beads for the detection of rare epigenetic events. Clin Chim Acta 425(0): 169-175.

Laboratories K (2013) Kyoto Encyclopedia of Genes and Genomes Vol. 2013. Available from: http://www.genome.jp/kegg/ (accessed on 2013).

Malmstrom A, Gronberg BH, Marosi C, Stupp R, Frappaz D, Schultz H, Abacioglu U, Tavelin B, Lhermitte B, Hegi ME, Rosell J, Henriksson R (2012) Temozolomide versus standard 6-week radiotherapy versus hypofractionated radiotherapy in patients older than 60 years with glioblastoma: the Nordic randomised, phase 3 trial. Lancet Oncol 13(9): 916-926.

Medvedeva YA, Khamis AM, Kulakovskiy, Ba-Alawi W, Bhuyan MS, Kawaji H, Lassmann T, Harbers M, Forrest AR, Bajic VB (2014) Effects of cytosine methylation on transcription factor binding sites. BMC Genomics 15: 119.

NICE (2011) The recognition and initial management of ovarian cancer, Clinical guideline 122: NICE. Available from: http://nice.org.uk/Guidance/ $\operatorname{cg} 122$.

Pomel C, Barton DP, McNeish I, Shepherd J (2008) A statement for extensive primary cytoreductive surgery in advanced ovarian cancer. BJOG 115(7): 808-810.

Sankaranarayanan R, Ferlay J (2006) Worldwide burden of gynaecological cancer: the size of the problem. Best Pract Res Clin Obstet Gynaecol 20(2): 207-225.

Shih KK, Chi DS (2010) Maximal cytoreductive effort in epithelial ovarian cancer surgery. J Gynecol Oncol 21(2): 75-80.

TCGARN (2011) Integrated genomic analyses of ovarian carcinoma. Nature 474(7353): 609-615.

Tothill RW, Tinker AV, George J, Brown R, Fox SB, Lade S, Johnson DS, Trivett MK, Etemadmoghadam D, Locandro B, Traficante N, Fereday S, Hung JA, Chiew YE, Haviv I, Gertig D, DeFazio A, Bowtell DD (2008) Novel molecular subtypes of serous and endometrioid ovarian cancer linked to clinical outcome. Clin Cancer Res 14(16): 5198-5208.

Vergote I, Trope CG, Amant F, Kristensen GB, Ehlen T, Johnson N, Verheijen RH, van der Burg ME, Lacave AJ, Panici PB, Kenter GG, Casado A, Mendiola C, Coens C, Verleye L, Stuart GC, Pecorelli S, Reed NS (2010) Neoadjuvant chemotherapy or primary surgery in stage IIIC or IV ovarian cancer. N Engl J Med 363(10): 943-953.

Wang C, Cicek MS, Charbonneau B, Kalli KR, Armasu SM, Larson MC, Konecny GE, Winterhoff B, Fan JB, Bibikova M, Chien J, Shridhar V, Block MS, Hartmann LC, Visscher DW, Cunningham JM, Knutson KL, Fridley BL, Goode EL (2014) Tumor hypomethylation at 6p21.3 associates with longer time to recurrence of high-grade serous epithelial ovarian cancer. Cancer Res 74(11): 3084-3091.

Wimberger P (2010) Influence of residual tumor on outcome in ovarian cancer patients with FIGO stage IV disease. Ann Surg Oncol 17(6): 1642-1648.

Yu L, Deng L, Li J, Zhang Y, Hu L (2013) The prognostic value of vascular endothelial growth factor in ovarian cancer: a systematic review and meta-analysis. Gynecol Oncol 128(2): 391-396.

Zhao D, Zhang F, Zhang W, He J, Zhao Y, Sun J (2013) Prognostic role of hormone receptors in ovarian cancer: a systematic review and metaanalysis. Int J Gynecol Cancer 23(1): 25-33.

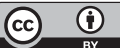

This work is licensed under the Creative Commons Attribution 4.0 International License. To view a copy of this license, visit http://creativecommons.org/licenses/by/4.0/

(C) The Author(s) named above 2017

Supplementary Information accompanies this paper on British Journal of Cancer website (http://www.nature.com/bjc) 